\title{
Provinzialismus und Semi-Intersektionalität: Fallstricke des Feminismus in postkolonialen Zeiten
}

Dieser Text entspringt einer Irritation, einem feministischen Disput, in den ich mich 2013 als Gastwissenschaftlerin am Centre of Excellence in Gender Studies der Quaid-i-Azam University verwickelt sah, einer renommierten staatlichen Hochschule in Islamabad. Ich hielt dort eine Reihe von Vorträgen, unter anderem über Intersektionalität. Dass diese Zugangsweise vom Rand des westlichen Feminismus in ihr Zentrum hat vordringen können (hooks 1984), hatte ich bis dahin für einen klaren Fortschritt gehalten: theoretisch, methodologisch und politisch. Farzana Bari, sozialistische Feministin, politische Aktivistin, öffentliche Intellektuelle und damals Direktorin des Centre, schätzte die Lage anders ein. Der intersektionalen Wende im westlichen Feminismus attestierte sie negative Effekte für ihre eigenen feministischen Aktivitäten vor Ort. Denn hinsichtlich des transnationalen Feminismus ging die intersektionale Wende mit der Problematisierung der Vorstellung eines globalen Patriarchats und der Schwesternschaft aller Frauen einher; besonders prononciert ausformuliert wurde dies im Kontext des postkolonialen Feminismus. Bari zufolge macht es diese Problematisierung zunehmend schwer, internationale Unterstützung für politische Kampagnen vor Ort zu finden. Bari ist nicht zuletzt im Zusammenhang von Ehrenmorden und anderen grundlegenden Frauenrechtsverletzungen eine wichtige feministische Stimme im öffentlichen Diskurs Pakistans.

Im Vergleich zu Deutschland ist Pakistan eine stark geschlechterdifferenzierte und -segregierte Gesellschaft (Haque 2010). ${ }^{1}$ Ursprünglich als säkularer Staat gegründet, ging das Land in den Jahren 1977 bis 1988 durch eine Phase der Militärdiktatur. In dieser Zeit fand eine umfassende

1 Ein anekdotisches Beispiel mag veranschaulichen, was dies mit Blick auf das universitäre Leben bedeutet. 2015 schloss das Girls' Hostel, das Studentinnenwohnheim auf dem Campus der QAU, abends um neun die Pforten - die Bewohnerinnen mussten dann zu Hause sein. Im Boys' Hostel gab es keine derartige Regel - die männlichen Studenten konnten kommen und gehen, wann sie wollten. Als ich einige von ihnen fragte, wie sie selbst eine abendliche Ausgangssperre fänden, kam die Antwort prompt: "We would break the door.» 
Restrukturierung von Staat und Gesellschaft statt, in deren Zuge auch Frauen und religiöse Minderheiten entrechtet wurden. Die eindeutig diskriminierenden Gesetze, die in jener Zeit verabschiedet wurden, kodierte und legitimierte die Regierung als "islamisch" (Fleschenberg 2010; Saigol 2016). Und auch wenn diese nach jahrelangen frauenpolitischen Protesten zum Teil wieder abgeschafft wurden, ist die rechtliche Lage für Frauen alles andere als günstig (Toor 2011, 159-184) und ist ein "military-mosque nexus", das heißt eine enge Verbindung zwischen Militär und religiösen Organisationen weiterhin Realität (Shaheed 2010, 854). Auch die Politisierung des Islam durch rechtsgerichtete Kräfte ist weiterhin Praxis - mit deutlich spürbaren Auswirkungen auf Alltag und politische Kultur, einschließlich der Marginalisierung säkularer Stimmen (Shaheed 2017, 115).

Dies bedeutet weder, dass der Islam als Religion auf rechtskonservative Positionen reduziert werden könnte oder sollte, noch, dass die pakistanische Geschlechterordnung von religiösen bzw. von politisch-religiösen Faktoren allein beeinflusst wäre. Pakistanische Sozialwissenschaftlerinnen führen die aktuelle geschlechterpolitische Lage vielmehr auf ein breites Bündel an Faktoren zurück. Hierzu zählten komplexe Machtkonfigurationen und -kämpfe im Zuge der unterschiedlichen jüngeren Stationen der pakistanischen Geschichte, namentlich Kolonialismus, Nationalismus, Diktatur, Demokratie und globaler Krieg gegen den Terror; aber auch die westliche Entwicklungszusammenarbeit - einschließlich Gender and Development - sowie die saudische Entwicklungspolitik, die unter anderem in der Finanzierung konservativer religiöser Schulen manifest wird, gelten als wichtige Einflussfaktoren (Khan / Zia 1995; Saigol 2016). Die pakistanische Geschlechterordnung wird hier also nicht nur auf endogene Ursachen zurückgeführt. Neben kulturellen Einflussfaktoren werden vielmehr verschiedene externe politische, ökonomische und auch politisch-religiöse Faktoren angeführt, nicht zuletzt die vielfältigen Effekte des letzten Afghanistankrieges, an dem auch Deutschland beteiligt war.

Im Folgenden geht es weniger um Pakistan als um Komplexitäten des Feminismus in postkolonialen Zeiten. Meine Erfahrungen vor Ort und die Diskussionen mit Farzana Bari erscheinen mir in diesem Zusammenhang aber wichtig; denn sie haben meine intersektionalen und postkolonialen Überzeugungen herausgefordert und die folgenden Überlegungen inspiriert. Seit Jahren gehe ich davon aus, dass der intersektionale Feminismus aus analytischen und aus politischen Gründen nicht-intersektionalen feministischen Positionen vorzuziehen ist. Denn letztere reflektieren häufig in erster Linie die Interessen und Prioritäten jener Frauen, denen in Bezug auf Merkmale wie "Rasse» und Ethnizität, Religion, Klasse oder 
Sexualität ein vergleichsweise privilegierter Status zukommt. Farzana Bari ist anderer Ansicht. Für sie, die lokal handelt, aber global denkt, stellt sich der intersektionale Feminismus praktisch als Entsolidarisierung dar, und zwar als Entsolidarisierung in einem Kampf, in dem vergleichsweise privilegierte Frauen ihre Privilegien just zu dem Zweck einsetzen, weniger privilegierte Frauen bei der Realisierung basaler Rechte zu unterstützen und damit zugleich in die öffentliche Verhandlung von Geschlechterverhältnissen zu intervenieren. Bari ist Aktivistin und verfolgt das Ziel, gravierende geschlechterpolitische Missstände zu bekämpfen. Dies erfordert mehr als eine kritische Analyse dieser Missstände. Und es erfordert vermutlich auch mehr als eine kritische Analyse der eigenen Privilegien.

Aber was genau ist es, das es erfordert, insbesondere seitens potentieller Verbündeter im Westen? Wie kann und sollte eine internationale und kontextübergreifende feministische Solidarität aussehen, die Baris Bedenken ernst nimmt, ohne dabei zentrale intersektionale und postkoloniale Einsichten aufzugeben? Dies sind die Fragen, die ich im Folgenden zu beantworten suche. $\mathrm{Zu}$ diesem Zweck gehe ich in fünf Schritten vor. $\mathrm{Zu}$ nächst erinnere ich an die Idee des globalen Feminismus sowie an die postkoloniale Kritik an diesem Konzept. Zweitens gehe ich auf das ein, was Farzana Bari als Effekt des Intersektionalitätsdiskurses beschreibt, nämlich eine Tendenz des gegenwärtigen kritischen feministischen Aktivismus im Westen, sich von transnationalem Engagement zurückzuhalten; diese Tendenz bezeichne ich als feministischen Provinzialismus. Drittens stehen Positionen der kritischen feministischen Theorie und Wissenschaft im Mittelpunkt, die der erwähnten Zurückhaltung entsprechen, und ich argumentiere, warum ich sie für nicht ausreichend intersektional halte, sondern eher als eine Form der Semi-Intersektionalität verstehe. Viertens geht es um weibliche Figuren des gegenwärtigen Orientalismus, die im Hintergrund der globalen Geschlechterpolitik eine Rolle spielen; dabei soll deutlich werden, dass auch die Zuschreibung von Handlungsfähigkeit keinesfalls vor Vereinnahmung und Instrumentalisierung schützt. Dem folgt fünftens ein kurzes Fazit, in dem ich Möglichkeiten umreiße, die sowohl bezogen auf die eher forschungsbezogenen Aspekte der transnationalen feministischen Solidarität als auch mit Blick auf feministischen Aktivismus Auswege aus den zuvor skizzierten Problemen versprechen könnten. 


\section{Globaler Feminismus}

Das Konzept des globalen Feminismus ist eng mit dem Konzept der globalen Schwesternschaft verbunden, das von der US-amerikanischen Wissenschaftlerin und Aktivistin Robin Morgan mit ihrem Buch Sisterhood is global verbreitet wurde (Morgan 1984). Dieses über 800 Seiten starke Kompendium erschien bereits Mitte der 1980er Jahre und damit deutlich vor dem digitalen Zeitalter mit seinen hervorragenden weltweiten Informationsmöglichkeiten. Es vereint 68 Länderkapitel - von Afghanistan bis Simbabwe - zur Situation von Frauen. Für jedes Land bietet es Informationen über die demografische Situation, über die Regierung und die politische Situation, über die Wirtschaft (weibliche Erwerbsbeteiligung, Gender Pay Gaps usw.), über die rechtliche Situation in Bezug auf Ehe, Scheidung, Familie, Soziales, Empfängnisverhütung, Abtreibung, Illegitimität, Homosexualität, Inzest, sexuelle Belästigung, Vergewaltigung, Gewalt, traditionelle / kulturelle Praktiken und Krisenzentren, über Frauengeschichte und über Mythologie; gefolgt von einem Artikel (in einigen Fällen auch von literarischen Texten oder Poesie) einer lokalen feministischen Aktivistin über die Situation der Frauen und der Frauenbewegung des jeweiligen Landes.

Laut Morgan ist das Buch von lokalen feministischen Kämpfen geprägt; sein Ziel besteht darin, diese Kämpfe zu vernetzen, und zwar ausgehend von der Prämisse, dass Frauen die unterdrückte Mehrheit sowohl fast aller Nationen als auch der menschlichen Spezies seien (Morgan 1984, 3). In ihrem ausführlichen Einleitungskapitel, in dem sie versucht, die Grundlagen für einen "planetarischen Feminismus« zu formulieren, argumentiert Morgan zu diesem Zweck in erster Linie vergleichend. Dennoch fällt auf, dass sie wesentlich mehr Interesse daran zeigt, gemeinsame GeschlechterMuster in den verschiedenen Ländern zu identifizieren als Unterschiede. Globale Schwesternschaft beschreibt sie als "cross-cultural, cross-agegroup, cross-occupation / class, cross-racial, cross-sexual-preference, and cross-ideological assemblage of women's voices" (Morgan 1984, 3). Sie identifiziert Einschränkungen reproduktiver Rechte, sexuelle Arbeitsteilung, geschlechtsspezifische Lohnunterschiede, Vergewaltigung, häusliche Gewalt, Pornografie und Prostitution als Probleme, mit denen Frauen weltweit konfrontiert sind. Vor diesem Hintergrund konstatiert - man kann auch sagen: konstruiert - sie eine weltweite Geschlechterasymmetrie mit einem übergreifenden Muster und lokalen Variationen. Morgans globaler Feminismus ist so gesehen ein globales, intern plurales Netzwerk ähnlicher feministischer Kämpfe, die globalgesellschaftlichen Wandel in 
großem Stil anstreben: "a complete social, political, economic, cultural, technological, sexual, and emotional transformation of human society" (Morgan 1984, 28). Es ist eine Form der transnationalen feministischen Solidarität, die Differenzen transzendiert und sich auf gemeinsame Probleme und Ziele konzentriert. Hinsichtlich geeigneter politischer Strategien für dieses Projekt schlägt Morgan ein Vorgehen vor, das Interventionen auf der jeweils nationalen Ebene und im UN-System sowie autonome Bewegungspolitik vereint.

Seit seiner Veröffentlichung wurden Morgans Buch wie auch das Konzept der globalen Schwesternschaft binnenfeministisch scharf kritisiert: vor allem dafür, Hierarchien und Machtunterschiede zwischen Frauen unsichtbar zu machen, da die Metapher der Schwesternschaft impliziere, alle Frauen säßen im selben Boot auf einem rauen Meer des Patriarchats und des Sexismus. Und tatsächlich identifiziert Morgan sehr klar Differenzen und Machtrelationen zwischen Männern und Frauen; doch sie schweigt über Differenzen und Machtrelationen zwischen verschiedenen globalen Kontexten, obwohl diese das Verhältnis zwischen Frauen mitunter maßgeblich beeinflussen (Wichterich 2007). Darüber hinaus de-thematisiert Morgan die historischen Prozesse, die regionale, nationale und lokale Geschlechterregime ins Leben gerufen haben - Prozesse, die oft viel mit Kolonialismus und Imperialismus und daher mit globalen Verbindungen statt nur mit lokalen Traditionen zu tun haben. Beispiele dafür gibt es viele - man denke nur an die Rolle katholischer Geschlechter- und Sexualitätsnormen in Lateinamerika, die Kodifizierung des Scharia-Rechts im kolonialen Indien oder die Feminisierung der Armut aufgrund von Strukturanpassungsmaßnahmen in vielen Teilen der Welt. Folgt man der postkolonialen Kritikerin Chandra Mohanty, erzeugt Morgan auf diese Weise sowohl ein empirisch falsches als auch ein politisch fragwürdiges Bild von Frauen als homogener Gruppe mit gemeinsamen Unterdrückungserfahrungen, einer geteilten epistemologischen Perspektive und geteilten politischen Zielen. Alternativ schlägt Mohanty vor, transnationale feministische Kooperationen im Sinne politischer Koalitionen zu verstehen. Das ist alles andere als ein organisches Modell wie jenes der Schwesternschaft. Vielmehr ist es ein strategisches Modell. Mohanty fasst transnationalen Feminismus als aus der Not geborene Kollaboration samt der für derartigen Kollaborationen typischen Kompromisse und möglichen Konflikte (Mohanty 1992). 


\section{Feministischer Provinzialismus}

Mohanty ist nicht die einzige geblieben, die westliche, d.h. im Westen bzw. im globalen Norden definierte Vorstellungen von globaler feministischer Solidarität dafür kritisiert hat, dass sie oftmals im Sinne feministischer Anliegen ausformuliert wurden, die just diesen westlichen Kontexten entstammen. Diverse andere Autorinnen haben ähnliche Kritiken vorgebracht, nicht zuletzt mit Blick auf die Institutionalisierung des globalen Feminismus westlichen Stils im Bereich der Entwicklungspolitik (Marchand/Parpart 1995; Saunders 2003). Diese Kritik läuft meist auf die These hinaus, dass sich die westliche globale feministische Solidarität, wenn sie praktisch und damit faktisch wird, häufig als Form von Imperialismus äußert. Kritische Feministinnen im Westen haben diese Problematisierung ernst genommen - und sich in der Konsequenz weitgehend von Versuchen der Realisierung globaler Schwesternschaft abgewandt. Damit haben sie - oder eher: haben wir - jedoch womöglich das Kind mit dem Bade ausgeschüttet. Denn wie Farzara Bari bereits vor Jahren monierte, haben wir uns in vielen Fällen von Nord-Süd-Solidarität und damit von der Sorge um globale Geschlechtergerechtigkeit insgesamt verabschiedet; mit dem Argument oder der Befürchtung, dass ein solches Engagement notwendigerweise eine imperiale Geste sei. Ich schlage vor, diese Position als feministischen Provinzialismus zu bezeichnen. Diesen feministischen Provinzialismus gilt es in meinen Augen zu problematisieren - und zwar nicht nur aus den politikstrategischen Gründen, auf die Farzana Bari hingewiesen hat, sondern auch aus einem normativen Grund. Angesichts der Tatsache, dass wir spätestens seit Beginn des europäischen Kolonialismus in einer vernetzten Welt leben und damit in einer Welt, die von Konstellationen geprägt ist, die zumindest partiell in globaler Geschichte und Politik wurzeln, sollte Solidarität niemals an nationalen oder anderen willkürlichen Grenzen enden. Der Umstand, dass sich das koloniale Projekt in vielen Fällen selbst als Solidarität in sehr weitem Sinne präsentiert hat, nämlich als zivilisatorische Mission und als Dienst an Fortschritt und Entwicklung, ändert daran ebenso wenig wie das Problem, dass Akte der Solidarität, die Statusdifferenzen überschreiten, vielleicht nie ganz davor gefeit sind, in Bevormundung umzuschlagen (Kerner 2018). Solche Faktoren mögen sowohl Konzeptionen als auch die Praxis von Solidarität schwieriger machen. Dadurch wird sie aber keinesfalls unnötig. Im Anschluss an Susan Buck-Morss plädiere ich dafür, die Grundkonstellation unserer widersprüchlichen, vielfältigen und doch gemeinsamen Welt, die von globalen Verwicklungen, Ko-Konstituierungen und Momenten der 
Überdetermination ebenso geprägt ist wie von unterschiedlichen Formen lokaler und nationaler Asymmetrien, Dissoziationen und Widerstandsmomente, als "globale Immanenz" zu verstehen - denn es gibt in dieser Konstellation kein Außerhalb. Nach Buck-Morss folgt aus der Annahme einer solchen Immanenz zum einen eine globale moralische Verantwortung, zum anderen die Aufgabe einer globalen und dabei doppelten Kritik (Buck-Morss 2003, 93, 96). Der Begriff der »doppelten Kritik« verdankt sich dem marokkanischen Soziologen Abdelkebir Khatibi, der ihn geprägt hat, um ein kritisches Verfahren vorzuschlagen, das zum einen koloniale bzw. postkoloniale Gesellschaften von innen kritisiert und zum anderen westliche Konzepte, die zur Beschreibung bzw. zur Konstruktion solcher Kontexte dienen, dekonstruiert - und in diesem Sinne etwa mit Blick auf Herrschaftsstrukturen im Maghreb sowohl die westliche Tradition des Marxismus als auch das theologische, charismatische und patriarchale Erbe der Region problematisiert und hinsichtlich seiner Grenzen befragt (Khatibi 2019, 26, 2). Khatibis Modell der doppelten Kritik entspringt einer spezifischen geo-politischen Position, und zwar einer kolonisierten und damit nicht-hegemonialen. Es versteht sich nicht von selbst und könnte diskutiert werden, ob und inwiefern dieses Modell verallgemeinert werden kann, ob es als Instrument der Kritik von jedweder geo-politischen Position in unserer verwobenen, aber dennoch stratifizierten Welt aus taugt, und ob es auch ausgehend von einer geo-politisch hegemonialen, beispielsweise einer westlichen Position Verwendung finden kann. Doch für Buck-Morss ist diese letztlich standpunkttheoretische Frage gar nicht wichtig. Vorrangig für das von ihr vorgeschlagene Projekt einer globalen Kritik ist nämlich nicht die Frage, wer sie vornimmt. Es handelt sich dabei sowieso um ein kollektives Projekt, das sich der unterschiedlichsten intellektuellen Ressourcen bedienen sollte, säkularer wie religiöser, moderner wie postmoderner. Vorranging für Buck-Morss ist vielmehr die Frage, wie diese Kritik aussieht (Buck-Morss 2003, 99). In diesem Zusammenhang plädiert sie für ein doppeltes Vorgehen im Sinne Khatibis - es geht darum, identitätspolitische Projekte in einem kosmopolitischen Sinne zu transzendieren und potentiell nichts von der Kritik auszunehmen (BuckMorss 2003, 101f.). Der linke Kosmopolitismus, der ihr dabei vorschwebt, geht mit einer Vorstellung sozialer Gerechtigkeit einher, die keine Gruppe der Menschheit von den Vorteilen und von der moralischen Verantwortlichkeit in der globalen Öffentlichkeit ausnimmt (Buck-Morss 2003, 102). Dieser Vorschlag scheint mir auch für den Bereich des Feminismus in postkolonialen Zeiten richtungweisend. Wenngleich die Frage, wie er umzusetzen ist, damit alles andere als beantwortet ist. 
Denn wenn der feministische Provinzialismus, eine Politik der vermeintlich sauberen Hände, die zudem die globale Immanenz bewusst oder unbewusst verkennt, keine gute Lösung ist, wie kann dann eine gute Lösung aussehen? Wem gegenüber sollten sich kritische westliche Feministinnen solidarisch zeigen, und wie? Und wie gehen wir bestmöglich mit dem komplexen Zusammenspiel von faktischen Differenzen und den unterschiedlichen Ebenen und Formen von Macht um? Mit den globalen Hierarchien, die im Verlauf der Geschichte des Kolonialismus und Imperialismus etabliert wurden, mit ungleich verteilten Rechten, Möglichkeiten und Privilegien, mit den subtilen und den weniger subtilen Machtwirkungen, die Kooperationen über faktischen Differenzen hinweg für gewöhnlich kennzeichnen, und mit den Machteffekten - manche würden sagen: der epistemischen Gewalt (Spivak 1988) - die Diskurse über diese Belange fast unweigerlich reproduzieren? Auch diese Fragen sind nicht leicht zu beantworten. Daher möchte ich zunächst versuchen zu erläutern, warum mir das, was derzeit als die common-sense Antwort des kritischen, intersektional und postkolonial informierten westlichen Feminismus erscheint, nicht reicht, oder eher: zu weit geht; und warum wir uns bemühen sollten, bessere Lösungen zu finden.

\section{Semi-Intersektionalität}

Während die aktivistische Reaktion auf die Kritik der globalen Schwesternschaft oftmals der skizzierte Provinzialismus war, die Konzentration auf Probleme vor der eigenen Haustüre, liefen die akademischen Reaktionen und Bearbeitungen auf wichtige Analysen und Problematisierungen der verschiedenen Varianten des Verhältnisses von Feminismus und Imperialismus hinaus. Aus dem intersektionalen und postkolonialen Blick auf Differenzen und Machtverhältnisse zwischen Frauen sind (allein schon im deutschen Sprachraum) Studien erwachsen zu westlichen Biases in der (transnationalen) feministischen Forschung und Praxis (u.a. Hess / Lenz 2001; Hacker 2012; Hostettler/Vögele 2014), zum Othering des Sexismus (u. a. Dietze / Brunner/Wenzel 2009) sowie zur langen Tradition der Instrumentalisierung der Frauenbefreiung für koloniale, imperiale und nationalistische Zwecke - also zu dem, was Gabriele Dietze zugespitzt "Ethnosexismus« nennt (Dietze 2017, $291 \mathrm{ff}$.). Die postkolonial informierte feministische Theorie fokussiert in diesem Sinne sowohl die rassistischen, nationalistischen und imperialen Sedimente im Feminismus selbst als auch die rassistischen, nationalistischen und imperialen Indienstnahmen fe- 
ministischer Rhetorik. Beides halte ich für überaus wichtig - und habe auch mit meinen eigenen Arbeiten dazu beizutragen versucht (u. a. Kerner 1999; 2005; 2010; 2012; 2016; 2017).

Dennoch vertrete ich im Folgenden die These, dass dabei Wichtiges auf der Strecke bleibt. Das ist zunächst die kritische Aufmerksamkeit für faktische Unterschiede zwischen den Geschlechterordnungen verschiedener Kontexte, einschließlich der Privilegien - vor allem in Form individueller Freiheiten in einem weiten Sinne -, die mit dem Leben in einem liberalen Rechts- und Wohlfahrtsstaat einhergehen, in dem politische Kämpfe für Geschlechtergerechtigkeit nennenswerte Erfolge für sich verbuchen konnten. Im kritischen westlichen Feminismus geht es viel um Privilegien, im Rahmen der Critical Whiteness Studies etwa um weiße Privilegien, zum Teil aber auch um Klassenprivilegien (Tißberger u.a. 2009; Eggers u. a. 2005; Kerner 2013). Diese Diskussion ist allerdings weitgehend von methodologischem Nationalismus bzw. Provinzialismus geprägt. Thematisiert werden unterschiedliche Privilegierungen innerhalb eines vergleichsweise eng umrissenen Kontextes, zumeist eines Nationalstaates. Doch die immensen Privilegien, die global gesehen mit einem "guten" Pass und einer "guten" Staatsbürgerschaft einhergehen, Privilegien, die von einer weitgehend visumsfreien globalen Freizügigkeit über eine passable Gesundheitsversorgung bis dahin reichen, auf der Grundlage sozialer Sicherungssysteme das Erwachsenenleben unabhängig von der eigenen Herkunftsfamilie gestalten zu können, werden weitgehend dethematisiert. Ayelet Shachar spricht im Zusammenhang des Zufalls, in welchen Kontext und in welche Staatsbürgerschaft wir jeweils ohne jedes eigene $\mathrm{Zu}$ tun hineingeboren werden, nicht ohne Grund von einer Birthright Lottery (Shachar 2009). Vor dem Hintergrund der globalen Immanenz ließe sich hoffen, dass aus den Privilegien des Lebens in einem vergleichsweise komfortablen Kontext eine Verpflichtung oder zumindest Bereitschaft erwachsen könnte, sich denjenigen gegenüber solidarisch zu zeigen, die sich in einer weniger komfortablen Lage wiederfinden und sich dort - oder anderswo - für positive Veränderungen einsetzen. Der postkolonial informierte westliche Feminismus geht allerdings eher in die andere Richtung. Eine Kritik von Sexismus und Patriarchat nicht lediglich vor der eigenen Haustüre, sondern auch anderswo, unterlässt er weitgehend.

In diesem Sinne wage ich die Behauptung, dass die Reaktion des kritischen westlichen Feminismus auf die Problematisierung der Idee einer globalen Schwesternschaft nicht intersektional genug ausfällt; und zwar weil sie sich vornehmlich mit Problemen von Rassismus, Nationalismus und Imperialismus im Zusammenhang von Feminismus und Geschlechterpoli- 
tik auseinandersetzt, und über dieser offenkundig wichtigen Aufgabe eine Kritik der vielfältigen Varianten des Sexismus, die unsere Welt ebenfalls prägen und die sich aus den unterschiedlichsten lokalen, nationalen, aber eben auch transnationalen Faktoren speisen, aus den Augen verliert. Eine Kritik von Sexismus anderswo, jenseits des je eigenen Kontextes, ist nicht nur aus der Mode gekommen, sondern gilt zudem oft als moralisch verdächtig - geht eine solche Kritik doch immer mit dem Risiko einher, eine imperiale Geste zu reproduzieren. Der Wunsch, dieses Risiko zu vermeiden, hat zu etwas geführt, das ich Semi-Intersektionalität nennen möchte. Dies soll an einem Beispiel illustriert werden.

In ihrem bemerkenswerten Aufsatz "Expanding the Combat Zone« umreißt die feministische Friedens- und Konfliktforscherin Claudia Brunner die Geschichte der Instrumentalisierung der Geschlechterpolitik für koloniale und imperiale Zwecke vom britischen Kolonialismus in Südasien bis heute; nach Brunner handelt es sich dabei um eine Geschichte vergeschlechtlichter und sexualisierter epistemischer Gewalt (Brunner 2016, 371). Im Anschluss an Gayatri Spivaks vielzitierte Beobachtung, dass diese Instrumentalisierung in ihren kolonialen Anfängen als Operation repräsentiert wurde, in der "[w]eiße Männer [...] braune Frauen vor braunen Männern" retten (Spivak 2008, 78), identifiziert Brunner vier ähnliche Logiken aus jüngerer Zeit. Neben dem "patriarchal genderism", ihrem Namen für die ursprüngliche, bereits von Spivak beschriebene Logik, ist das erstens der "embedded feminism" (weiße Frauen retten braune Frauen vor braunen Männern), zweitens der "transnational sexism" (weiße und braune Frauen retten braune Frauen vor braunen Männern), drittens der "homonationalism" (weiße Queers retten braune Queers vor braunen Männern) sowie schließlich "queer imperialism" (braune Queers retten braune Queers vor braunen Männern) (Brunner 2016, 380). Bedeutend an dieser Liste ist der auch von Brunner selbst betonte Umstand, dass in den von ihr unterschiedenen Logiken zwar sowohl die Rettenden als auch die zu Rettenden variieren, diejenigen aber, die als Gefahr erscheinen, immer die Gleichen sind: braune Männer.

Die Tendenz der Studie, alles, was auf dem Terrain der transnationalen Geschlechterpolitik geschieht, letztlich als Variation derselben kolonialen Ur-Logik zu interpretieren, birgt jedoch nicht nur Vorteile. Dies wird besonders deutlich im Zusammenhang dessen, was Brunner transnationalen Sexismus nennt. Sie übernimmt diese Bezeichnung von Robin Lee Riley, um zu problematisieren, dass anti-islamische Feminismen und Genderdiskurse zur Rechtfertigung US-geführter Militärinterventionen herangezogen werden (Brunner 2016, 376) - eine Problematisierung, der ich nur 
zustimmen kann. Weiterhin moniert Brunner jedoch, dass Frauen of Color - ihre Beispiele sind Waris Dirie und Ayan Hirsi Ali - oder besser gesagt die feministische Kultur- und Religionskritik ausgewählter Frauen of Color von liberalen feministischen Einstellungen und Praktiken angeeignet würde; diese Einstellungen und Praktiken wiederum nennt sie quasi in einem Atemzug mit okzidentalistischen Diskursen und einem "new racism that is about empire building and therefore in need of people of color as tokens or iconic figures in order to silence dissent (Brunner 2016, 376). Später in ihrem Text betont Brunner, dass epistemische Gewalt nicht nur eine Praxis des Othering sei, sondern auch eine des "Selving" (Brunner 2016, 382) - womit sie Strategien des imperialen Machterhalts durch die Hervorbringung einer besonders positiven europäischen bzw. westlichen Identität meint (Brunner 2016, 384). Ich möchte noch einmal bekräftigen, dass ich Brunners Analyse für richtungsweisend und wichtig halte; dennoch sollte man sie daraufhin überprüfen, was sie ihrerseits de-thematisiert, und welche nicht-intendierten Effekte sie produziert. In meinen Augen bringt sie die feministische Kultur- und Religionskritik von Frauen of Color zum Schweigen. Brunner diskutiert diese nicht als Beiträge zu einem transnationalen Kampf gegen die unterschiedlichen Varianten von Patriarchat und Sexismus, sondern ausschließlich als Alibipositionen im Dienste okzidentalistischer Akte epistemischer Gewalt. ${ }^{2}$

In einer Diskussion über feministische Umgangsweisen mit dem sogenannten Krieg gegen den Terror, die 2013 in der Zeitschrift Critical Studies on Terrorism erschienen ist, hat die in Großbritannien lehrende Politikforscherin Nicola Pratt Schweigen als angemessenes Strategie im kritischen Umgang mit just jenen Logiken geadelt, die Brunner kritisiert. "From the position of academia in the European and North American metropoles," erklärt Pratt, "perhaps the only way to avoid becoming complicit in racialising discourses and co-opted in the pursuit of the swar on terror', is to be strategically silent about 'women's rights` per se and, instead, adopt a feminism that resists processes of militarisation and neo-liberalisation that dehumanize and dispossess people« (Pratt 2013, 330). In meinen Augen opfert Pratt Frauenrechte und einen wirklich intersektionalen Feminismus,

2 Für ein weiteres Beispiel einer solchen Thematisierung - oder eher De-Thematisierung - der geschlechterpolitischen Belange von liberal argumentierenden Frauen und Feministinnen of Color zugunsten einer kritischen Thematisierung kolonialer Sedimente im westlichen Feminismus vgl. die ansonsten überzeugende Abhandlung von Mendel und Neuhold (2015, 43); für eine Kritik dieser Tendenz anhand einer Diskussion von Joan Scotts Überlegungen zum französischen Kopftuchverbot vgl. Abbas (2014, 47-50). 
der alle seine Sektionen jederzeit ernst nimmt, auf dem Altar einer Politik der sauberen Hände. Das strategische Schweigen, das sie vorschlägt, hat zum Ziel, sich der Vereinnahmbarkeit durch die falsche Seite zu entziehen. Mögliche weitere Konsequenzen - etwa negative Konsequenzen für jene, denen das strategische Schweigen eigentlich zugute kommen soll thematisiert Pratt leider nicht.

Afiya Shehrbano Zia, feministische Aktivistin und Wissenschaftlerin in Pakistan, hat die Konsequenzen von Pratts Position für die Erfolgsaussichten feministischer Kämpfe in Konfliktkontexten entsprechend scharf kritisiert. Die Idee, dass Feministinnen von einer Priorisierung von Gender-Belangen abrücken sollen, so temporär und so strategisch motiviert dies auch sein mag, bedeutet für Zia eine Priorisierung patriarchaler Politik, oder, in ihren eigenen Worten, "that patriarchal politics must be prioritized, especially in times of conflict" (Zia 2013b, 333). Vor dem Hintergrund des jahrzehntelangen feministischen Widerstands in Pakistan gegen "this same demand of silence by religious / conservative nationalists who accuse us of washing our dirty laundry in front of western eyes" (Zia 2013b, 332) führt Pratts Idee Zias Einschätzung nach in die falsche Richtung.

Darüber hinaus problematisiert Zia das Tokenism-Argument, das wir von Brunner kennen. Zia zufolge läuft dieses Argument nämlich entgegen seinen Intentionen just auf eine Verkennung nicht-hegemonialer Positionen und Autorinnen hinaus - denn es unterstelle Feministinnen in und aus Konfliktregionen, die sich aktiv für Geschlechtergerechtigkeit einsetzen, eine Komplizinnenschaft mit imperialen Kriegen und Gewalt. In Zias Augen stellt diese Unterstellung eine problematische Form der Rassialisierung dar - "as if brown women do not know themselves or blindly follow the dictates of white feminist agendas" (Zia 2013b, 332).

Dass die Stimmen nicht-westlicher säkularer Feministinnen, die eine kritische Haltung gegenüber der jeweils hegemonialen Kultur und politisierten Religion ihres Herkunfts- bzw. Alltagskontextes einnehmen, im postkolonial inspirierten kritischen westlichen Feminismus nicht grade populär sind, ist allein schon deshalb bemerkenswert, weil die meisten postkolonial inspirierten kritischen westlichen Feministinnen mit Bezug auf die hegemoniale Kultur und die politisierten Religionen ihrer eigenen Herkunfts- und Alltagskontexte sehr wohl eine kritische Position einzunehmen beanspruchen. Man könnte daraus schlussfolgern, dass hier mit zweierlei Maß gemessen wird. Dies allerdings ist eine koloniale Praxis par excellence. 


\section{Figuren des Orientalismus der Gegenwart}

Die Literaturwissenschaftlerin Sadia Abbas vertritt die These, dass die muslimische Figur, die der liberale westliche Diskurs verlangt, nicht (mehr) der heroische Autor ist wie Salman Rushdie - man könnte hier auch Waris Dirie oder Ayan Hirsi Ali nennen - sondern vielmehr die fromme Muslima the "pious, Muslim woman" (Abbas 2014, 5). Saba Mahmood hatte bereits 2005 eine in der Folge äußerst einflussreiche ethnographische Studie mit dem Titel Politics of Piety vorgelegt (Mahmood 2005); in und mit diesem Buch schrieb Mahmood gegen die von ihr konstatierte okzidentalistische einschließlich liberalfeministische - Tendenz der Viktimisierung muslimischer Frauen an, und zwar indem sie die Handlungsfähigkeit konservativer islamischer Aktivistinnen in Kairo aufzeigte. Abbas zufolge schreibt der liberale westliche Diskurs der Figur der frommen Muslima Handlungsfähigkeit - auf englisch Agency - inzwischen zu, und zwar nicht zuletzt, um damit die eigene Liberalität, die eigene Toleranz gegenüber religiös Andersdenkenden zu unterstreichen. Die Figur der frommen Muslima wird dabei - eigentlich ganz im Sinne Mahmoods - zu jener der agentialen muslimischen Frau (Abbas 2014, 5). Nur am Rande sei erwähnt, dass diese Figur zwar überaus gegenwärtig, dabei jedoch alles andere als einheitlich konnotiert ist. Bezieht sich Brunner auf sie, assoziiert sie diese Figur mit säkularen muslimischen Feministinnen (Brunner 2016, 377). Abbas hingegen assoziiert die Figur - wie übrigens auch Zia (2013a) - mit Akteurinnen, die sich klar vom (säkularen) Feminismus abgrenzen und die - zumindest in Pakistan - eher zu den politischen Gegnerinnen von (säkularen) Feministinnen zählen als zu ihren Verbündeten: Frauen, die in Abbas Worten Erfüllung in konsensualer - und mithin agentialer - Selbstunterwerfung finden, anstatt für gleiche Rechte zu kämpfen; und die ihre Handlungsfähigkeit in den Dienst eines konservativen politisch-religiösen Programms stellen, das Geschlechterdualismen und -asymmetrien stützt (Abbas 2014, 85; vgl. auch Jamal 2012, 110; Zia 2009).

Abbas vertritt ferner die These, dass die Figur der handlungsfähigen frommen Muslima und die uns ebenfalls bereits bekannte Figur des weiblichen muslimischen Opfers, das es vor braunen Männern zu retten gilt, als zwar deutlich distinkte, doch zugleich komplementäre politische Rechtfertigungsfiguren fungieren. Denn beide Figuren laden dazu ein, in konservativen politischen Programmatiken im Zusammenhang von Imperialismus und Anti-Imperialismus instrumentalisiert zu werden. "So if Laura Bush [...] could offer the vision of female suffering under (say) the Taliban as a justification for war, the anti-imperialists can, if they choose, 
cite Islamist women who loathe the burdens of modernity« schreibt Abbas (Abbas 2014, 85). Für sie führt dies zu einer hochproblematischen Homogenisierung des Islam, der hier in erster Linie als anti-modern erscheint, sowie der Heterogenität von Frauen in islamischen und muslimisch geprägten Staaten, Gesellschaften und Kontexten, unter denen sich freilich wie überall sonst auf der Welt - alle möglichen Positionen finden lassen, von säkular bis ultra-religiös, und die diese Positionen mit einer großen Bandbreite von Praktiken verknüpfen; Praktiken, von denen einige aus westlicher Perspektive westlich erscheinen mögen, andere eindeutig nichtwestlich (Abbas 2014, 55). Abbas zufolge führen beide Figuren, die der agentialen frommen Muslima und die des weiblichen muslimischen Opfers, darüber hinaus zu einer Dichotomisierung der muslimischen Welt auf der einen Seite und des Westens auf der anderen - eine Dichotomisierung, die muslimische Frauen einmal mehr als das Andere des Westens erscheinen lässt (Abbas 2014, 88). Abbas zufolge reproduziert dies orientalisti-

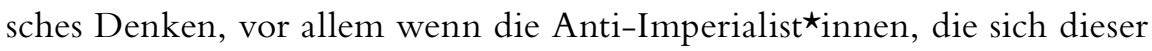
Dichotomisierung bedienen, kritische westliche Anti-Imperialist ${ }^{\star}$ innen sind (Abbas 2014, 48f.). Ferner trage die Fokussierung auf die skizzierten Figuren dazu bei, Sexismus zu dethematisieren. "White men may be trying to save brown women from brown men", schreibt Abbas; "but brown men may indeed oppress brown women, and brown women (elite and otherwise) may also collude in sustaining structures of misogyny (Abbas 2014, 71). Wenn man den zweiten Teil dieses Satzes genauso ernst zu nehmen gedenkt wie den ersten, ist die Anerkennung der agentialen frommen Muslima bei weitem keine hinreichende feministische Strategie. Wie Buck-Morss spricht sich auch Abbas für eine doppelte Kritik aus - diese bedeutet für sie, »that feminist concern - within colonized, postcolonial, decolonized, reimperiled cultures, diasporas, societies, nations - cannot be postponed« (Abbas 2014, 71).

\section{Fazit}

Feminismus und Gleichstellungsdiskurse werden für rechte Projekte wie Rassismus, Nationalismus und Imperialismus instrumentalisiert; und solche Formen der Instrumentalisierung müssen kritisiert werden. Aber auch Kultur und Religion werden für rechte Projekte instrumentalisiert; und die Verteidigung ungleicher Geschlechterverhältnisse spielt dabei in der Regel eine wichtige Rolle. Der Feminismus sollte dies weder ignorieren, noch strategisch dazu schweigen, noch sich scheuen, dagegen anzu- 
kämpfen. Und in unserer verflochtenen Welt gilt dies unabhängig vom jeweiligen Standort. Dass grade die globale Geschlechterpolitik durch die genannten Indienstnahmen und Instrumentalisierungen noch komplexer geworden ist, als sie im Lichte intersektionaler und postkolonialer Kritiken ohnehin schon erschien, ist zwar misslich, aber wohl kaum zu ändern.

Was also gilt es zu tun, und wie? Für die feministischen Wissenschaften, die Diskursproduktion, gilt es erstens, angesichts der umrissenen Komplexitäten den Sexismus nicht aus den Augen zu verlieren - und zwar nirgendwo. Dieser sollte dann allerdings weder als weltumspannende Einheitslogik mit lokalen Variationen verstanden werden, noch als allein kulturellen oder religiösen Faktoren geschuldet. Vielmehr sind seine Geschichte, seine Reproduktion, seine Umkämpftheit und dabei entstehende Verschiebungen und Brüche zu untersuchen - Kontext für Kontext. Dabei sollten auch globale Faktoren, vom Imperialismus über den Kapitalismus bis hin zu entwicklungspolitischen Einflussnahmen Berücksichtigung finden: das westliche Gender and Development ebenso wie der "donor-driven Islam«, d.h. die affirmative Bezugnahme westlicher entwicklungspolitischer Organisationen auf religiöse Traditionen und Institutionen zu Ungunsten lokaler säkularer feministischer Positionen und Akteur`innen (Zia 2011), sowie saudische Initiativen.

Zweitens ist jede Essentialisierung von Kultur und Religion ebenso zu vermeiden wie die Reproduktion kolonialer Denkweisen, vor allem die Dichotomisierung von Okzident und Orient, wobei ja traditionellerweise ersterer als freiheitlich, säkular und demokratisch dargestellt wird (ungeachtet von Phänomenen wie Kolonialismus, Nationalsozialismus, Rechtspopulismus, Rassismus, und christlichem Fundamentalismus, aber auch schon von Staatskirchen) und letzterer als rückschrittlich, despotisch und irrational (Said 2009). Die Dinge sind komplizierter als derartige Reduktionismen. Dies liegt nicht zuletzt daran, dass der europäische Kolonialismus und der westliche Imperialismus seit jeher zwar nicht ausschließlich, aber zumindest auch einen Export just dessen darstellen, was Europa und der Westen selbst grade nicht zu sein beanspruchen - und daher allzu gern als ihr originär Anderes präsentieren. Sogenannte schlechte Regierungsführung in ehemaligen Kolonien bzw. Postkolonien (Mbembe 2001) ist hierfür ein besonders trauriges, aber daher auch besonders gutes Beispiel.

Drittens schließlich ist eine Gleichsetzung von Islam und Unterdrückung zu vermeiden. Frauen- und familienpolitischer Konservatismus und dualisierte Geschlechterbilder florieren in diversen Religionen - auch der Katholizismus und Teile des Protestantismus bieten hier besten Nährboden. Den Sexismus und den Antifeminismus, der in fundamentalistischen 
Strömungen von Religionen verbreitet wird, gilt es dabei jedoch ebenso ernst zu nehmen wie die politische Indienstnahme von Religionen durch rechtsgerichtete Kräfte, die auf den ersten Blick nicht als besonders religiös erscheinen. Im pakistanischen Feminismus ist der global vernetzte, geschlechterpolitisch regressive religiöse Fundamentalismus seit längerem Thema. Das macht den pakistanischen Feminismus - bzw. seine auf Formen des Fundamentalismus und extremen Konservatismus konzentrierte politische Kultur- und Religionskritik - zur globalen Avantgarde. In westlichen Länder werden regressive geschlechterpolitische Manifestationen religiöser Player und Institutionen, in diesem Fall vor allem katholischer und evangelikaler Provenienz, erst in jüngster Zeit explizit zum Thema (Hark / Villa 2015). Hier besteht einiger Nachholbedarf, nicht zuletzt mit Blick auf die globalen Vernetzungsaktivitäten rechtsgerichteter politischer und politisch-religiöser Gruppierungen.

Für die praktische Arbeit im Feld der globalen Geschlechterpolitik gilt es wohl zunächst, deren Agenden im Rahmen transversaler Dialoge zu bestimmen und nicht in den europäischen Head-Offices entwicklungspolitischer Firmen, Behörden und Projekte, wie dies bis heute oft geschieht. Aber auch dann wird die Sache vermutlich trotzdem nicht mit dauerhaft sauberen Händen zu haben sein. Feminismus ist komplex, zumal in postkolonialen Zeiten. ${ }^{3}$

\section{Literatur}

Abbas, Sadia (2014): At Freedom's Limit. Islam and the Postcolonial Predicament. New York.

Brunner, Claudia (2016): Expanding the Combat Zone. In: International Feminist Journal of Politics 18 [Nr. 3], 371-389.

Buck-Morss, Susan (2003): Thinking Past Terror. Islamism and Critical Theory on the Left. London - New York.

3 Die erste Version dieses Textes entstand für die Tagung "Hermeneutic Conflict, Cultural Entanglement, and Social Inequality. Power of Interpretation in Intersectional Perspective« im September 2016 in Rostock; spätere Fassungen konnte ich 2017 bei der "European Conference on Politics and Gender" in Lausanne und beim "Forschungskolloquium Transkulturelle Geschlechterforschung" in Bielefeld vortragen und 2018 mit dem "Geneva Political Theory Colloquium» und im Rahmen der Ringvorlesung "Geschlechterdimensionen von Macht und Herrschaft" in Bonn dis-

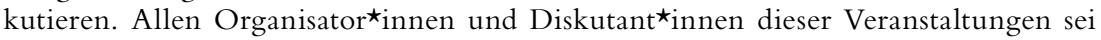
hiermit herzlich gedankt. Mein Dank für wichtige Impulse und Hinweise gilt ferner Farzana Bari, Andrea Fleschenberg und den Herausgeberinnen dieses Heftes. 
Dietze, Gabriele (2017): Sexualpolititk. Verflechtungen von Race und Gender. Frankfurt - New York.

Dietze, Gabriele / Brunner, Claudia / Wenzel, Edith (Hrsg.) (2009): Kritik des Okzidentalismus. Transdisziplinäre Beiträge zu (Neo-)Orientialismus und Geschlecht. Bielefeld.

Eggers, Maureen Maisha/Kilomba, Grada/Piesche, Peggy/Arndt, Susan (2005): Mythen, Masken und Subjekte: Kritische Weißseinsforschung in Deutschland. Münster.

Fleschenberg, Andrea (2010): Military Rule, Religious Fundamentalism, Women's Empowerment and Feminism in Pakistan. In: Roces, Mina / Edwards, Louise (Hrsg.): Women's Movements in Asia: Feminism and Transnational Activism. London - New York, $166-188$.

Hacker, Hanna (2012): Queer entwickeln. Feministische und postkoloniale Analysen. Wien.

Haque, Riffat (2010): Gender and Nexus of Purdah Culture in Public Policy. In: South Asian Studies 25 [Nr. 2], 303-310.

Hark, Sabine /Villa, Paula-Irene (Hrsg.) (2015): Anti-Genderismus. Sexualität und Geschlecht als Schauplätze aktueller politischer Auseinandersetzungen. Bielefeld.

Hess, Sabine/Lenz, Ramona (Hrsg.) (2001): Geschlecht und Globalisierung. Ein kulturwissenschaftlicher Streifzug durch transnationale Räume. Königstein / Taunus.

hooks, bell (1984): Feminist Theory. From Margin to Center. Boston.

Hostettler, Karin/Vögele, Sophie (Hrsg.) (2014): Diesseits der imperialen Geschlechterordnung. (Post-)koloniale Reflexionen über den Westen. Bielefeld.

Jamal, Amina (2012): Feminism and "Fundamentalism« in Pakistan. In: Tahir, Madiha R./ Memon, Qalandar Bux/Prashad, Vijay (Hrsg.): Dispatches from Pakistan. Minneapolis, $104-120$.

Kerner, Ina (1999): Feminismus, Entwicklungszusammenarbeit und postkoloniale Kritik. Eine Analyse von Grundkonzepten des Gender-and-Development Ansatzes. Hamburg.

Kerner, Ina (2005): Forschung jenseits von Schwesternschaft. Zu Feminismus, postkolonialen Theorien und Critical Whiteness Studies. In: Harders, Cilja / Kahlert, Heike/Schindler, Delia (Hrsg.): Forschungsfeld Politik. Geschlechtskategoriale Einfürung in die Sozialwissenschaften. Wiesbaden, 217-238.

Kerner, Ina (2010): Verhält sich intersektional zu lokal wie postkolonial zu global? Zur Relation von postkolonialen Studien und Intersektionalitätsforschung. In: Reuter, Julia / Villa, Paula-Irene (Hrsg.): Postkoloniale Soziologie. Empirische Befunde, theoretische Anschlüsse, politische Intervention. Bielefeld, 237-258.

Kerner, Ina (2012): Varianten feministischer Kritik. In: Birkle, Carmen/Kahl, Ramona/ Ludwig, Gundula/Maurer, Susanne (Hrsg.): Emanzipation und feministische Politiken: Verwicklungen, Verwandlungen, Verwerfungen. Sulzbach, 51-65.

Kerner, Ina (2013): Critical Whiteness Studies: Potentiale und Grenzen eines wissenspolitischen Projekts. In: Feministische Studien 31 [Nr. 2], 278-293.

Kerner, Ina (2016): Transnationalismus, Geschlecht und Intersektionalität. Bausteine einer Feministischen Internationalen Politischen Theorie. In: Kreide, Regina / Niederberger, Andreas (Hrsg.): Internationale Politische Theorie. Umrisse und Perspektiven eines neuen Forschungsfeldes. Stuttgart, 155-169.

Kerner, Ina (2017): Relations of difference: Power and inequality in intersectional and postcolonial feminist theories. In: Current Sociology Review 65 [Nr. 6].

Kerner, Ina (2018): Solidarity across Difference Lines. In: Social Politics 25 [Nr. 1], 44-49. Khan, Nighat Said/Zia, Afiya Shehrbano (Hrsg.) (1995): Unveiling the Issues: Pakistani Women's Perspectives on Social, Political and Ideological Issues. Lahore. 
Khatibi, Abdelkebir (2019): Plural Maghreb. Writings in Postcolonialism. London

Mahmood, Saba (2005): Politics of Piety: The Islamic Revival and the Feminist Subject. Princeton.

Marchand, Marianne/Parpart, Jane L. (1995): Feminism/Postmodernism/Development. London - New York.

Mbembe, Achille (2001): On the Postcolony. Berkeley - Los Angeles.

Mendel, Iris / Neuholdt, Petra (2015): Feminismus und Antirassismus - another unhappy marriage? Der Diskurs um `kulturelle Gewalt und die Möglichkeiten transnationaler feministischer Solidarität. In: Feministische Studien 33 [Nr. 1], 38-54.

Mohanty, Chandra Talpade (1992): Feminist Encounters: Locating the Politics of Experience. In: Barrett, Michèle / Phillips, Anne (Hrsg.): Destabilizing Theory. Contemporary Feminist Debates. Stanford, 74-92.

Morgan, Robin (1984): Sisterhood is Gobal: The International Women's Movement Anthology. Garden City.

Pratt, Nicola (2013): Weaponising feminism for the "war on terror", versus employing strategic silence. In: Critical Studies on Terrorism 6 [Nr. 2], 327-331.

Said, Edward (2009): Orientalismus. Frankfurt/M.

Saigol, Rubina (2016): Feminism and Women's Movements in Pakistan. Islamabad.

Saunders, Kriemild (2003): Feminist Post-Development Thought: Rethinking Modernity, Post-Colonialism and Representation. London.

Shachar, Ayelet (2009): The Birthright Lottery: Citizenship and Global Inequality. Cambridge, MA.

Shaheed, Farida (2010): Contested Identities: gendered politics, gendered religion in Pakistan. In: Third World Quaterly 31 [Nr. 6], 851-867.

Shaheed, Farida (2017): Pakistan's Women's Movement. Protests, Programming, and Revitalization. In: Basu, Amrita (Hrsg.): Women's Movements in the Global Era: the Power of Local Feminisms. Coulder, 95-128.

Spivak, Gayatri Chakravorty (1988): Can the Subaltern Speak? In: Nelson, Cary/Grossberg, Lawrence (Hrsg.): Marxism and the Interpretation of Culture. Urbana - Chicago, $272-313$.

Spivak, Gayatri Chakravorty (2008): Can the Subaltern Speak? Postkolonialität und subalterne Artikulation. Wien.

Tißberger, Martina/Dietze, Gabriele/Hrzán, Daniela/Husmann-Kastein, Jana (Hrsg.) (2009): Weiß - Weißsein - Whiteness: Kritische Studien zu Gender und Rassismus. Frankfurt/M. u.a.

Toor, Saadia (2011): The State of Islam. Culture and War Politics in Pakistan. London.

Wichterich, Christa: Transnationale Frauenbewegungen und Global Governance. In: gender...politik...online (2007). https://www.fu-berlin.de/sites/gpo/int_bez/globalisie rung/Transnationale_Frauenbewegungen/wichterich.pdf (07.08.2019)

Zia, Afiya Shehrbano (2009): The Reinvention of Feminism in Pakistan. In: Feminist Review 91 [Nr.], 29-46.

Zia, Afiya Shehrbano: Donor-driven Islam. In: Open Democracy (2011). https://www. opendemocracy.net/en/5050/donor-driven-islam/ (07.08.2019)

$\mathrm{Zia}$, Afiya Sherhbano (2013a): Redefining faith and freedoms: the "war on terror« and $\mathrm{Pa}-$ kistani women. In: Critical Studies on Terrorism 6 [Nr. 2], 323-326.

Zia, Afiya Sherhbano (2013b): A response to Nicola Pratt's call for »strategic silence«. In: Critical Studies on Terrorism 6 [Nr. 2], 332-333. 